\title{
El idioma inglés en el currículo universitario: importancia, retos y alcances
}

\section{The English Language in University Curriculum: Importance, Challenges and Achievements}

\author{
Carolina España Chavarría \\ Escuela de Secretariado Profesional \\ Universidad Nacional de Costa Rica \\ Heredia, Costa Rica \\ cespanac@yahoo.com.mx
}

Recibido 07 de agosto de 2009 • Aceptado 14 de setiembre de 2009

\begin{abstract}
Resumen. La importancia de la inclusión del idioma inglés en el currículo universitario está basada en las exigencias de la globalización e intercambios de mercado, por lo que se cree necesaria la formación del idioma inglés en los estudiantes como futuros profesionales que contribuirán al desarrollo integral de la nación.
\end{abstract}

Palabras clave. Globalización, mundialización, revolución tecnológica, currículo, inglés, segunda lengua.

Abstract. The importance of English language inclusion into university's curriculum is based on the demands of globalization and market interchanges, that is why English language must be considered as part of students' instruction as future professionals that will contribute to the integral development of the nation.

Key words. Globalization, mundialization, technological revolution, curriculum, English, second language.

Las recientes transformaciones sociales y económicas evidenciadas en este último siglo han demandado de la universidad del siglo XXI un replanteamiento de sus funciones, exigiéndole nuevas formas de entender y gestionar el conocimiento. Es decir, el conocimiento, las experiencias, la sed de aprender, entender y la necesidad de hacer, obligan a la universidad a transformase y reinventarse como el espacio de reflexión, cultivo y procesamiento del conocimiento y experiencias por excelencia.

\footnotetext{
Estudiante del Doctorado en Políticas y Prácticas de Innovación Educativa para la Sociedad del Conocimiento por la Universidad de Almería, España. Magíster en Políticas y Prácticas de Innovación Educativa para la Sociedad del Conocimiento por la Universidad de Almería, España. Magíster en Segundas Lenguas y Culturas con énfasis en Inglés como Lengua Extranjera para: Alumnado Adulto, por la Universidad Nacional, Costa Rica. Docente en el área de la educación y enseñanza del inglés. Actualmente, labora en la Escuela de Secretariado Profesional de la Universidad Nacional, Costa Rica. Cuenta con ponencias y asesorías internacionales realizadas en la Universidad de San Carlos, Guatemala (USAC): Técnicas para la comunicación oral en inglés orientada a la locución, estrategias y metodologías de la enseñanza del idioma inglés, Técnicas para la comunicación oral en inglés orientada al periodismo, Profesionalización del colectivo docente de la Escuela de Linguística.
} 
Los desafíos de la educación contemporánea impactan las propuestas curriculares promovidas y demandan su transformación al margen de lo que la actual sociedad del conocimiento requiere.

Por ello, a la hora de definir el término currículo universitario es posible entenderlo como la planificación sistemática de todo aquello a lo que el proyecto educativo le da valor formativo. Se constituye en plataforma de medición intelectual e interpreta las necesidades de la sociedad para convertirlas en objetivos de aprendizaje. Además, dicha planeación articula los objetivos establecidos con los contenidos, procesos, recursos, entre otros de los elementos que la conforman y, asimismo, traza el camino formativo que llevará al estudiantado al desarrollo de sus competencias, a la luz de los requerimientos de la sociedad a la que debe responder y atender.

De allí que el currículo universitario hoy más que nunca le otorgue protagonismo a la formación en un segundo idioma, caso específico del inglés, valorado como un ingrediente clave y de demanda creciente por la omnipresente globalización y revolución tecnológica que nos envuelve; expresión misma del sistema económico y social del mundo occidental contemporáneo, los procesos de modernización y su vinculación con los medios de difusión de masas, y a la incidencia de estos procesos en la sociedad industrializada, la cual influye en el desarrollo educativo, social, económico y político de nuestros países.

Las exigencias profesionales y personales que impone este mundo globalizado fuerzan a la universidad a ponerle especial atención a la formación de profesionales bilingües; por cuanto en la actualidad el manejo de un segundo idioma, como es el inglés, pasó de ser un valor agregado a constituirse en una competencia lingüística que requiere desarrollar el individuo para integrarse con efectividad y eficacia a la nueva concepción de mundo, definida por la UNESCO como mundialización-entendida esta como el resultado de una transformación social y, consecuentemente, educativa-, impactada por la evidente apertura de mercados y el desarrollo tecnológico producto de la globalización.

La vinculación del inglés al currículo educativo podría concebirse como parte de las nuevas preocupaciones y exigencias de la mundialización; sin embargo, dicha vinculación debe concebirse como un elemento más de la formación integral del estudiantado, no solo a nivel linguiístico y cultural, sino también crítico, reflexivo, activo y creador y, por sobre todo, deberá nacer en el seno mismo de la planificación curricular y pedagógica. Es decir, el aprendizaje del idioma inglés no puede supeditarse a las demandas del mercado, sino que debe constituir parte de las intenciones de formación que la universidad misma se imponga.

Por lo tanto y siendo este un siglo determinado por la globalización y la calidad en la educación (esta última no solo una simple mercancía, sino una parte central de una cultura y una sociedad), el aprendizaje del inglés complementa de forma integral la formación profesional y se concibe como una de las exigencias del mercado laboral más evidentes, junto con el manejo de los recursos tecnológicos. Al respecto, el Ph.D. Olman Segura, ex rector de la Universidad Nacional de Costa Rica, afirmó:

(...) la inclusión de cursos de inglés y de tecnologías de informática en el currículo de las diferentes carreras es parte de esta internacionalización a la que se dirige la Universidad Nacional, tomando en cuenta que en el mundo globalizado actual el manejo de este idioma, de uso universal, y el adecuado uso de las tecnologías de la información y la comunicación son herramientas esenciales que requieren todos los y las profesionales (sic) para vincularse exitosamente al mercado laboral. (Plan Global Institucional, 2004, p. 5)

Coincidiendo con Segura, el manejo del idioma inglés es uno de los pilares del currículo universitario, este a su vez es valorado como herramienta de información y comunicación y su 
apropiado manejo, por parte de quienes se forman y forman en dicha institución, es fundamental para competir en el actual mundo laboral y personal.

Además, es una responsabilidad social universitaria desarrollar su proyecto de universidad de la mano del proyecto de país, reinventando su currículo por uno de carácter integrador, flexible, socialmente armónico, resolutivo, actual, significativo, que promueva el empoderamiento del conocimiento; que motive la puesta en marcha de iniciativas de innovación, y que incite mediante la formación; pero sobre todo que favorezca el uso de la competencia lingüística (caso específico del idioma inglés); la interrelación de saberes; la comunicación con seres que hablen o escriban en otros idiomas; el compartir percepciones culturales y de mundo de forma más directa y vivencial, entre otros.

De allí que la vinculación del idioma inglés con el resto de los saberes considerados por la universidad debe estar reflejado en las políticas y prácticas educativas, con el fin de garantizar una formación integral, efectiva y eficaz para el estudiantado.

Por tanto, si el inglés no es vinculado con significancia en los procesos de formación del estudiantado universitario, futuros profesionales y motores claves del desarrollo socioeconómico de este país, entonces, podría estarse atentando contra el proceso de internacionalización al que pretende dirigirse, en este caso específico, la Universidad Nacional y, a su vez, se estaría poniendo en riesgo la formación integral y proactiva que busca y requiere el colectivo estudiantil universitario.

Así, vale la pena preguntarse si el dominio de un segundo idioma supone la adquisición de nuevas competencias y conocimientos que contribuyen al manejo de nuevas tecnologías y a la capacidad de adaptación y comunicación que este sistema demanda para enfrentar eficientemente la imponente globalización. De ser así, es posible suponer, tal y como lo sugiere Pérez y Sola (2004), que el bilingüismo esté ligado al éxito educativo, laboral y social, que de forma reiterada pregona la mundialización, en congruencia con lo que buscan los miembros de la sociedad actual.

El dominio del idioma inglés supone ser un elemento clave en la lista compleja de nuevos conocimientos, habilidades, competencias y destrezas que un profesional de este milenio supone poseer para ser competitivo en el mercado laboral, como también considera Freire (2002), parte del currículo globalizado e interdisciplinario del cual hoy se habla y se pretende implementar. De ahí que los centros de educación universitaria estén llamados a nutrir su currículo enlazando al idioma inglés con el resto de los elementos que lo integran y hacen de este modelo de planificación de la formación universitaria el camino que conduce a atender las actuales exigencias de la sociedad del conocimiento.

Hoy en día es evidente que el manejo de una sola lengua no es suficiente para responder a las demandas que impone un mundo interconectado, el cual ve impactadas las relaciones de trabajo, estudio y convivencia por el idioma. De allí la necesidad del desarrollo de la capacidad y competencia lingüística de los futuros profesionales, con el fin de que dicho colectivo tenga una visión pluralista del mundo; entre en contacto, de forma enérgica y efectiva, con otras maneras de expresión y pensamiento, y se facilite el reconocimiento de la tolerancia, respeto y aceptación de los unos con los otros.

Los centros universitarios enfrentan el reto de formación lingüística que le garantice al grupo discente su incorporación y permanencia en el mercado laboral, en donde se supone los nuevos profesionales han de desarrollar tareas tanto sencillas como también complejas, posiblemente relacionadas al manejo de idiomas requeridos en el mundo mercantil.

Asimismo, es posible considerar las demandas del sector productivo, liderado en su mayoría por multinacionales o empresas de capital estadounidense y dedicadas a la inversión y relación comercial, a las cuales, sin profesionales bilingües, les podría ser más difícil el desarrollo y permanencia en el país, con lo que se estaría corriendo el riesgo de perder fuentes de empleo, si dichas compañías optasen por trasladarse a mercados multilingües. 
Aunado a lo anterior y como marco referencial de las relaciones comerciales vigentes a nivel nacional, es posible creer que Estados Unidos de América es uno de los socios comerciales de Costa Rica de mayor importancia. Por consiguiente, la necesidad de incorporar el inglés como una herramienta lingüística de intercambio comercial y cultural para la comunicación y promoción de aprendizajes, que aseguren conocimientos significativos en la formación profesional del estudiantado, significa un reto complejo; pero eminentemente necesario, si se busca el desarrollo integral colectivo e individual de una nación.

El conocimiento de una segunda lengua está estrechamente ligado a las necesidades de formación profesional que el educando tiene, así como a la eficacia y efectividad que su vinculación supone en el marco de la apertura de mercados inmersos en el fenómeno de la globalización. Ambos fenómenos sociales han sido parte de la expresión del desarrollo social y económico del mundo actual, y han tomado un protagonismo significativo en el mercado laboral de la sociedad costarricense. Tal y como lo apuntan las políticas curriculares expuestas por la UNA, las cuales establecen que cada plan de estudios deberá “(...) actualizarse sistemáticamente, a partir de estudios recientes que muestren las necesidades de los actores sociales involucrados, así como del entorno socioeconómico y cultural nacional y regional, para garantizar su pertinencia y oportunidad" (Plan Global Institucional de la 2004-2011, 2004, p. 5).

Estas exigencias o formas modernas que moldean el desarrollo mundial, denominadas por González (2001) como Globalización de la economía y mundialización de la cultura de origen neoliberal, no son más que una muestra clara del reordenamiento económico, al que Costa Rica se ve sometida y de lo cual la educación debe garantizar la integralidad y significancia del proceso que le atañe. Por esto estas formas deberían influenciar y determinar las actuales reformas educativas (metodología y/o estrategias didácticas) y las transformaciones curriculares en el quehacer universitario, ya que "El problema de la educación no es filosófico o axiológico sino técnico, la educación no debe ocuparse de los fines, pues los acepta tal y como ellos se nos dan; de lo que debe ocuparse es de los medios, de la técnica, para lograr la mayor eficacia en la consecución de los fines ya dados" (Ordóñez, 2001, p. 28).

Si la constante para evidenciar la eficacia y efectividad de la acción educativa gira entorno a los medios y técnicas implementados para la consecución de los fines ya dados, la vinculación del inglés a las políticas y prácticas curriculares universitarias es un elemento clave para la consecución de dichos fines.

Entonces, es posible pensar que la formación académico-profesional está requiriendo hoy más que nunca del desarrollo de competencias lingüísticas que faciliten una efectiva comunicación, concebida desde el dominio de una segunda lengua, elemento clave para contar con un perfil de profesional que este mundo globalizado requiere y espera, advirtiendo que "la comunicación acompaña esta nueva concepción de la empresa donde el hombre es el capital más precioso; la comunicación es el testigo y el agente de un nuevo contrato social" (Weil, 1992, p. 8).

Es evidente que el bilingüismo es requisito fundamental para una formación de calidad, en donde la plurifuncionalidad del profesional sea evidencia de su formación integral, y resulte un profesional proactivo, quien responda de forma crítica, analítica e investigativa a los múltiples retos laborales de la sociedad actual. Se facilita, así, el acceso al mercado laboral, orientado hacia la empresa privada generalmente multinacional, como también a las demandas de la sociedad de la información cada vez más influenciadas por el idioma y la cultura anglosajona.

Además de los beneficios laborales que el bilingüismo supone, este podría jugar un papel determinante en la transmisión cultural, la cual se constituye en uno más de los derechos que tiene el estudiantado de recibir una educación libre, significativa y funcional que sea vinculante con el eje 
de estudio para que se constituya en parte de su eje de interés y nutra de relevancia los aprendizajes promovidos (Blanco, 2006).

De allí la importancia del diseño de estrategias adecuadas para contrarrestar la pérdida de identidad cultural que el bilingüismo y la cibercultura pudiesen provocar en los procesos de formación que enfrenta el estudiantado. Es decir, quien logre desarrollar su competencia lingüística y hacer uso de una segunda lengua para efectos laborales o personales, no solo requerirá de dicho canal de comunicación, sino también deberá desarrollar asertividad cultural para lograr una comunicación efectiva.

Por lo tanto, si la práctica pedagógica que se adopta no busca la integración de aprendizajes, entonces el dominio práctico del inglés podría estar siendo inalcanzable para el estudiantado, quien se expone a más horas contacto con asignaturas técnicas que lingüísticas, reiterando así la carencia de integralidad y efectividad que el proceso educativo supone y evidenciando el hecho de que la vinculación del inglés con las demás disciplinas que conforman la malla curricular en las asignaturas impartidas en las titulaciones ofrecidas no está clara y podría, entonces, no ser de interés formativo.

La vinculación del inglés al currículo universitario es la respuesta para muchos futuros profesionales deseosos de triunfo laboral y que, por razones de condición social y económica, no pudieron contar con una formación bilingüe desde temprana edad.

Así como el mundo ha evolucionado y la globalización ha penetrado en la sociedad costarricense, así también, es posible creer que la percepción del bilingüismo como parte de una formación elitista ha dejado de ser la premisa. Por el contrario, nos hace pensar en el hecho de que este se ha convertido, tal y como se ha mencionado anteriormente, en una exigencia de mercado y un ingrediente clave para minimizar las brechas de acceso laboral en un mercado donde su moneda de cambio lingüístico es el inglés y lo que el individuo es capaz de ofrecerle al mercado debe estar fortalecido por el acceso al aprendizaje de una segunda lengua.

Las transformaciones curriculares en la educación de los últimos años, lideradas por el bilingüismo y las nuevas tecnologías de comunicación, han impactado -quizás no tan agresivamente como se debería- el currículo de las ofertas académicas universitarias -como es el caso de la UNA-, en donde prácticamente todas las carreras que allí se ofrecen cuentan con al menos dos niveles de inglés. Lastimosamente, en su mayoría de conocimiento instrumental del idioma (se avocan a formar al estudiantado para que sea capaz de entender textos escritos en el idioma inglés, sin necesidad siquiera de que este reporte lo entendido en el idioma meta), pese a las múltiples y ya mencionadas necesidades de formación que al respecto tiene la sociedad del conocimiento.

Asimismo, la necesidad de que la universidad le presta la debida atención a la formación bilingüe del estudiantado de forma sistemática y desde que empieza su formación universitaria obedece al hecho de que el estudiantado, en muchas ocasiones, comienza a trabajar desde que inicia su carrera profesional, momento en el cual la formación de competencias mal llamadas complementarias (lenguaje extranjero o computacional) están posicionadas muy adelante en los programas de estudio o, peor aún, se pueden llevar cuando se quiera, puesto que no son requisito para comprender otros saberes; pero, a su vez, suponen ser lo suficientemente útiles para afrontar el requisito lingüístico que el mercado laboral exige.

De allí que los aprendizajes promovidos en los primeros años de la carrera y que nutren la formación profesional de los estudiantes podrían ser un gancho de ingreso al mercado laboral, sin ser esta la única competencia requerida para lograr permanencia en el entorno laboral de este colectivo.

La necesidad de que la universidad replantee de forma enérgica el currículo que la distingue, y el cual da vida a su función formadora, ayudará a que el estudiantado no se prive más de poder desarrollar aprendizajes que requieran del entendimiento y uso de una segunda lengua, pues, por 
lo visto, no basta con que se reconozca la existencia de la necesidad, sino es necesario hacer una lectura de la calidad de la formación que este colectivo estudiantil y docente recibe para asumir tan complejo reto.

Por tanto, la puesta en marcha de un currículo integral y sostenible permitirá la formación de estudiantes que, con iniciativa, dinamismo y fortaleza, den respuesta a los múltiples retos que impone el mundo competitivo circundante; y permitirá que el estudiantado sea capaz de sostener un crecimiento económico de largo alcance, y contribuir con el mejoramiento social del país y con el mejoramiento en la calidad de vida de su persona y la de los demás.

El desarrollo social y económico de un país está estrechamente ligado a las políticas y prácticas educativas que se definan, de allí que quienes deseen incorporarse a la fuerza laboral en cualquier disciplina universitaria ofertada y reciban una educación en donde se conjuguen los saberes disciplinares con la formación en un segundo idioma, contarán con un perfil competitivo, producto de una visión curricular integral que el actual desarrollo socioeconómico demanda, exige y requiere para enfrentar los embates de la globalización.

Por tanto, es necesario concebir el inglés como una herramienta lingüística y cultural para la comunicación y promoción del conocimiento, herramienta que deberá estar contemplada en las políticas curriculares de la universidad y planteada de forma integral con respecto a las otras asignaturas a cursar en cualquier oferta académica, abriendo paso a la interdisciplinaridad de la acción educativa. Además, para lograr llevar a la realidad educativa universitaria la inclusión del inglés al currículo promovido, Cortés y Cabrera (2003) consideran necesario definir los criterios pedagógicos y tipos de aprendizaje promovidos en relación con dicho idioma, tales como el aprendizaje cooperativo, el aprendizaje basado en problemas o el método del caso, considerados metodologías activas que promueven el desarrollo integral del alumnado en áreas tales como la lingüística, interpersonal, investigativa y experimental.

Es posible concluir afirmando que la universidad está llamada a establecer políticas curriculares y orientaciones pedagógicas que evidencien la importancia de la integración del inglés en la promoción de aprendizajes y su incidencia en la calidad de formación y educación promovida, es decir, la universidad y sus artífices deberán tener un planteamiento claro en cuanto a ¿por qué? y ¿para qué? se imparte inglés en el sistema de educación universitaria y su implicación en el desarrollo socioeconómico del país. Más allá de una inclusión curricular, deberá existir pertinencia y vinculación interdisciplinaria del inglés como parte esencial de la integralidad del conocimiento que impone la mundialización, y que requiere el país para enfrentar los embates de la globalización y de la era de la información, y lograr, así, así el desarrollo requerido.

\section{Referencias biblográficas}

Blanco, N. (2006). Saber para vivir. En A. M. Piussi y A. Mañeru. (Coords.). Educación, nombre común femenino (pp. 158-183). Barcelona: Octaedro.

Cortés, A. y Cabrera, L. (2003). Programa de Inglés para la Conversación Área Comercial y Servicios Secretariado X, XI y XII. Costa Rica: MEP (Educación Técnica Profesional).

Freire, P. (2002). Pedagogía de la Autonomía. Argentina: Ediciones Siglo XXI. 
González, C. (2001). Reforma educativa y transformación curricular. Cuadernos Pedagógicos 3 del Ministerio de Educación de Guatemala, 1-55.

Ordóñez, J., (2001). La globalización de la tecnología, humanismo y educación. Globalización, identidades y educación. Cuadernos Pedagógicos 4 del Ministerio de Educación de Guatemala, 1-69.

Pérez, A. y Sola, M. (2004). Investigación e Innovación en la formación del Profesorado. España: Universidad de Málaga.

Plan Global Institucional 2004-2011. (2004). Análisis del Contexto Externo e Interno. Costa Rica: Universidad Nacional.

Weil, P. (1992). La comunicación global. Comunicación institucional y de gestión. Madrid: Pirámide. 